\title{
Overexpression of HSD17B4 exerts tumor suppressive function in adrenocortical carcinoma and is not associated with hormone excess
}

\author{
Guanxiong Ding ${ }^{1,2, *}$, Shenghua Liu ${ }^{1,2, *}$, Qiang Ding ${ }^{1,2}$ and Chenchen Feng ${ }^{1,2}$ \\ ${ }^{1}$ Department of Urology, Huashan Hospital, Fudan University, Shanghai 200040, PR China \\ ${ }^{2}$ Fudan Institute of Urology, Fudan University, Shanghai 200040, PR China \\ *These authors contributed equally to this work \\ Correspondence to: Chenchen Feng, email: drfengchenchen@163.com
}

Keywords: adrenocortical carcinoma; HSD17B4; tumor suppressor; p53

Received: January 25, $2017 \quad$ Accepted: November 07, $2017 \quad$ Published: December 01, 2017

Copyright: Ding et al. This is an open-access article distributed under the terms of the Creative Commons Attribution License 3.0 (CC BY 3.0), which permits unrestricted use, distribution, and reproduction in any medium, provided the original author and source are credited.

\section{ABSTRACT}

Aim: Adrenocortical carcinoma (ACC) is characterized with excessive hormone production. We therefore investigated expression of hormone-related genes in ACC.

Results: We queried status of $\mathbf{1 4}$ key genes directly involved in adrenal hormone production and found HSD17B4 expression was upregulated in 39\% of ACC cases on top of all queried genes. Overexpression of HSD17B4 was significantly associate with a normo-hormonal phenotype. Constitutive HSD17B4 expression was higher in ACC cell line NCI-H295R than in adrenocortical small cell carcinoma cell line SW13. NCI-H295R cells with HSD17B4-knockdown (KD) demonstrated significantly inhibited proliferation, increased apoptosis, and increased cell cycle arrest. Enrichment analysis for mRNA expression in ACC samples with or without HSD17B4 overexpression showed significant change in p53 pathway. Replenish of HSD17B4 in SW13 cells and knockdown of HSD17B4 in H295R cells confirmed alterations in MDM4, ATR, and IE24 with alterations more contrasting in H295R cells. HSD17B4-KD inhibited cell invasion, migration and anchorage independent growth of NCI-H295R cells, but not of SW13 cells.

Materials and Methods: Clinical and genetic data of ACC samples were reproduced from the ACC dataset of The Cancer Genome Atlas (TCGA) database using cBioPortal. Genes participating in adrenal hormone production were queried. Association between gene status and hormone release were studied and in vitro assays using RNA interference were carried out.

Conclusions: Overexpression of HSD17B4 exerted tumor suppressive function in adrenocortical carcinoma and was not related to hormone excess. Crosstalk between HSD17B4 and p53 warrants further investigation.

\section{INTRODUCTION}

Adrenocortical carcinoma (ACC) is a rare endocrine malignance at a prevalence of approximately 0.7 to 2 per million people $[1,2]$. While the disease is surgically resectable at earlier stages, it is substantially aggressive at advanced stages. Multidisciplinary modalities including chemotherapy, radiotherapy, surgical intervention and adrenolytic therapy are the mainstay of treatments for patients with stage III and IV diseases, which above all confer a poor prognosis [3-5].

The cortex of adrenal gland secretes a variety of vital hormones which are produced in excessive amount in ACC and causes severe symptoms. Starting from cholesterol, the adrenal cortex produces cortisol, mineralocortisol, and sex hormones in general under meticulous mediation from the central-peripheral axis via series of enzymes. In the malignant setting, dysregulated hormone excess could lead 
to disrupted balance in metabolism, electrolyte equilibrium and sexual appearance. Cortisol excess, the predominant type of hormone dysregulation in ACC, may substantially compromise immune response, allow viral replication, and bring cortisol-related multi-systematic complications, all being severe health problems [6-8]. However, whether excessive hormone secretion is intrinsically procarcinogenic or stands solely as a byproduct facilitating ACC to a minor extent, remains unclear. The answer lies in part in the contribution of genes encoding those enzymes for hormone production as well as in cases without hormone excess, which occur in approximately $40 \%$ of cases [9].

In the current study, we aim to systematically investigate the alterations of genes encoding hormone related genes in adrenal gland and their associations with clinical hormone phenotypes, as well as their genetic relevance to other oncogenic pathways in a relatively large cohort (over 90 cases) for this rare disease, thanks to The Cancer Genome Atlas (TCGA) project, which contains comprehensive and multilevel genetic information [10]. We expect to reveal roles of such genes other than hormonal regulation and provide novel insights on the potential to develop targeted strategy in ACC.

\section{RESULTS}

\section{HSD17B4 is overexpressed in ACC}

There were 14 genes encoding enzymes that participate in adrenal hormone production included in the current study, namely, CYP21A2, CYP11B1, CYP11B2, HSD3B1 HSD3B2, HSD3B7, CYP17A1, CYP21A2, CYP11B1, CYP11A1, CYP19A1, HSD17B1, HSD17B2, and HSD17B4. In general, alteration in at least one of the genes occurred in $66 \%$ of cases (61out of 92). Changes at expression level was predominant compared with mutation and CNV (Figure 1A). Of note, HSD17B4 was altered in $\sim 39 \%$ of cases, ranking top of all genes with overexpression in 29 cases, mutation in 3 cases and gene amplification in 1 case, respectively. We then evaluated the association between mRNA expression and hormone type and found that enhanced function of the genes was mainstay of alteration (Figure 1A-1B). Over half of the patients had excessive hormone release and cortisol was the major type (Figure 1B). Overexpression of HSD17B4 was not associated with excessive hormone release $(P=$ 0.668 , Figure 1B). In further analysis with breakdown of hormone types, association between HSD17B4 expression level and a certain or combination of hormones were not noticed either ( $P>0.05$ for all comparisons, Figure 1C). We then studied expression of each of the 14 genes and the association with hormone production and found that when regarded as a categorical variable, overexpression of HSD3B7 and HSD17B4 were associated with normohormonal phenotype while overexpression of CYP11A1 was associated with hormone excess (Table 1). However, there was no significant association detected when expression was analyzed as continuous variable (Figure 1D). In the univariate analysis for association between HSD17B4 expression and clinicopathological parameters, we found lack of significant association for adjuvant mitotane therapy $(P=0.092)$, tumor stage $(P=0.148)$, metastasis $(P=0.661)$, Weiss score $(P=0.143)$, response to primary therapy $(P=0.183)$, residual tumor status ( $P$ $=0.236)$, lymph node involvement $(P=0.578)$ or patient gender $(P=0.489)$. Tumors without necrosis showed lower HSD17B4 expression and the difference was close to statistical significance $(P=0.059)$. Patients with HSD17B4 overexpression had on average $\sim 50$ days longer overall survival than patients without. However, the difference did not reach statistical significance $(P=0.699$, Figure 1E).

\section{HSD17B4 knockdown promotes ACC growth}

Constitutive level of HSD17B4 was studied in 2 cell lines, in which NCI-H295R is ACC cells and SW13 is small cell carcinoma of adrenal cortex. NCIH295R cells showed substantially higher HSD27B4 level (Figure 2A). Genetic knockdown (KD) using shRNA efficiently downregulated HSD27B4 expression in both cells (Figure 2B). Adenovirus-mediated overexpression of HSD17B4 in both cells also showed high efficiency (Figure 2C). HSD27B4-KD NCI-H295R cells showed significantly increased proliferation, whilst the effect was not significant in SW13 cells (Figure 2D). Overexpression and KD of HSD17B4 induced significant increase and decrease in G1 population, respectively (Figure 2E). However, only HSD17B4 overexpression induced increased G1 population in SW13 cells and KD did not, indicating an inherent difference between the cancer types (Figure 2E). HSD17B4-KD also resulted in significant inhibition of cell apoptosis in NCI-H295R cells but not in SW13 cells (Figure 2F).

\section{p53 signaling is enriched in HSD17B4 upregulated cases}

The enrichment analysis yielded 1923 genes with expressions significantly enriched in cases with HSD17B4 overexpression. Further functional annotation revealed 77 significantly altered pathways annotated by KEGG PATHWAY database. Nine genes within p53 signaling showed significant enrichment in expression levels, including higher expressions of RCHY1, SIAH1, ATR and lower expressions of RFWD2, SHISA5, TNFRSF10B, EI24, TP53I3, and MDM4, respectively in HSD17B4 overexpressed cases (Figure 3A-3C). In validation study at translation level, we found that HSD17B4 KD in NCI-H295R cells showed higher MDM4, IE24 and lower ATR levels (Figure 3D). In SW13 cells, HSD17B4 overexpression showed the similar alteration in MDM4, IE24, and ATR levels (Figure 3E). 
Table 1: A Chi-square analysis of overexpression of hormone producing genes and presence of excessive hormone, reproduced from TCGA ACC dataset

\begin{tabular}{|c|c|c|c|c|}
\hline- & - & \multicolumn{2}{|c|}{ Excessive Hormone } & \multirow{2}{*}{$P$ value } \\
\hline - & - & - & + & \\
\hline \multirow{2}{*}{ CYP21A2 } & - & 31 & 49 & \multirow{2}{*}{0.1170} \\
\hline & + & 0 & 4 & \\
\hline \multirow{2}{*}{ CYP11B1 } & - & 26 & 47 & \multirow{2}{*}{0.5285} \\
\hline & + & 5 & 6 & \\
\hline \multirow{2}{*}{ CYP11B2 } & - & 29 & 48 & \multirow{2}{*}{0.6332} \\
\hline & + & 2 & 5 & \\
\hline \multirow{2}{*}{ HSD3B1 } & - & 31 & 50 & \multirow{2}{*}{0.1773} \\
\hline & + & 0 & 3 & \\
\hline \multirow{2}{*}{ HSD3B2 } & - & 31 & 47 & \multirow{2}{*}{0.0519} \\
\hline & + & 0 & 6 & \\
\hline \multirow{2}{*}{ HSD3B7 } & - & 24 & 50 & \multirow{2}{*}{0.0208} \\
\hline & + & 7 & 3 & \\
\hline \multirow{2}{*}{ CYP17A1 } & - & 31 & 47 & \multirow{2}{*}{0.0519} \\
\hline & + & 0 & 6 & \\
\hline \multirow[t]{2}{*}{ CYP21A2 } & - & 31 & 49 & \multirow{2}{*}{0.1170} \\
\hline & + & 0 & 4 & \\
\hline \multirow[t]{2}{*}{ CYP11B1 } & - & 26 & 47 & \multirow{2}{*}{0.5285} \\
\hline & + & 5 & 6 & \\
\hline \multirow[t]{2}{*}{ CYP11A1 } & - & 31 & 44 & \multirow{2}{*}{0.0152} \\
\hline & + & 0 & 9 & \\
\hline \multirow[t]{2}{*}{ CYP19A1 } & - & 29 & 45 & \multirow{2}{*}{0.2379} \\
\hline & + & 2 & 8 & \\
\hline \multirow[t]{2}{*}{ HSD17B1 } & - & 29 & 48 & \multirow{2}{*}{0.6332} \\
\hline & + & 2 & 5 & \\
\hline HSD17B2 & - & 30 & 52 & \multirow{2}{*}{0.6977} \\
\hline \multirow{3}{*}{ HSD17B4 } & + & 1 & 1 & \\
\hline & - & 9 & 29 & \multirow{2}{*}{0.0225} \\
\hline & + & 22 & 24 & \\
\hline
\end{tabular}

HSD17B4 is associated in part with metastatic potential and tumorigenesis

Both epithelial-mesenchymal transition (EMT) and anchorage-independent growth were indicators for metastatic potential. We found that HSD17B4-KD NCIH295R cells showed significantly increased cell invasion and migration (Figure 4A-4B). Also, HSD17B4-KD NCIH295R cells demonstrated significantly reduced colony formation (Figure 4C). Nonetheless, SW13 cells did not show any significant alteration in cell invasion, migration, or colony formation regardless of overexpression or $\mathrm{KD}$ (Figure 4A-4C). In the in vivo study, we observed significantly increased tumor growth in NCI-H295R cell with HSD17B4 KD (Figure 4D).

\section{DISCUSSION}

In the current study, we found that hormone related gene HSD17B4 exerted tumor suppressive function and was not associated with hormonal phenotype of ACC. To date, our study was the first to study a relatively complete panel of adrenocortical hormone related genes in ACC and the role of HSD17B4 therein was not previously reported. While it was not surprising that overexpression was the predominant genetic alteration of those genes given 
that $\sim 60 \%$ of ACC patients suffer excessive hormonal symptoms, it was astonishing the most frequently altered gene was not associated with any excessive hormone type. The heatmap however showed an obvious association between overexpression of certain genes (i.e. HSD3B2, CYP17A1, CYP11A1, and CYP19A1) and excessive hormone. In the further statistical analysis using a categorical stratification, in which overexpression of a certain gene was strictly defined, we find that HSD17B4 was significantly associated with normo-hormonal phenotype, indicating that HSD17B4 overexpression in ACC could be responsive to combat excessive hormone production and appears quite effective. Given that excessive hormone is immunosuppressive and the effect of HSD17B4 can be, in a way, tumor suppressive by modulating hormone level. This also corresponds with the previous reports that key genes for steroidogenesis are CYP subtypes and there could be functional redundancy for the rest of genes [1114]. Excess of hormone could bring hirsutism in female, gynecomastia in male, or Cushing syndrome that are substantially detrimental [15]. However, there lacks a clear association between excessive hormone with worsened or better prognosis [16]. It is thus possible that hormonal excess is a byproduct of ACC due to the inherent function of the adrenal gland parallel to tumorigenesis. This notion was further supported by the indication for mitotane, which serves as an antihormonal agent rather than antitumoral, implying separate regulatory pathways therein [17].

Overexpression of HSD17B4 is ubiquitous and in peripheral organs it plays a theoretical role in converting estradiol to estrone and $\mathrm{D}^{5}$-androstenediol to dehydroepiandrogen (DHEA), and both of which are supposed increase estrone. Given that adrenal glands provide precursors for target organ to produce terminal hormones, the lack of association between HSD17B4 and hormone in $\mathrm{ACC}$ can be attributed to tropism. However, carcinogenic role of HSD17B4 has been reported in other non-endocrine malignancies. Overexpression of HSD17B4 was noted in prostate cancer with significant association with worsened prognosis via possibly steroid metabolism and fatty acid oxidation [18]. In hepatocellular carcinoma, HSD17B4 was shown to act as an adaptor between inflammation and cancer cell proliferation [19]. In our study, however, HSD17B4 appears to play a
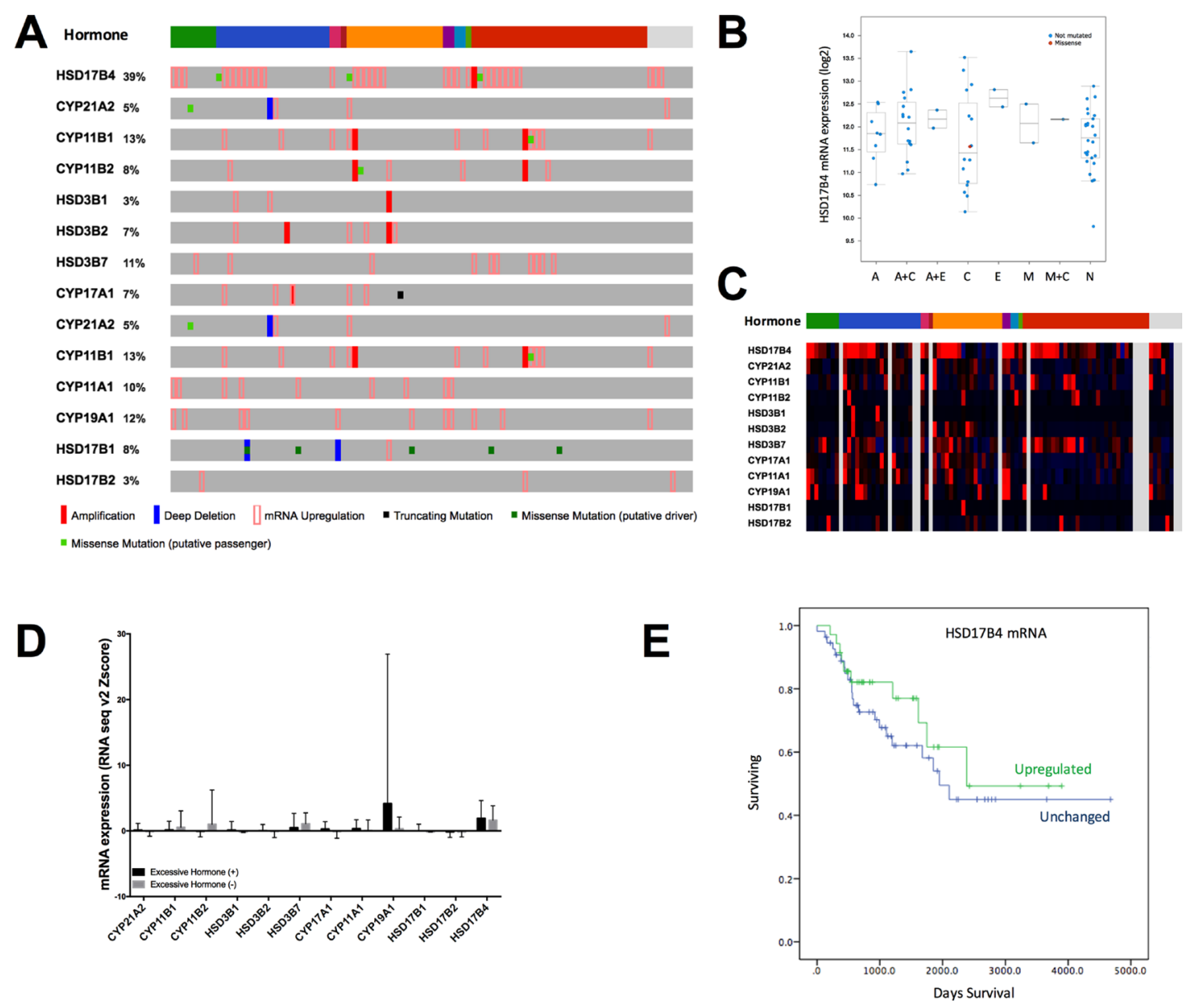

Figure 1: HSD17B4 is over expressed in adrenocortical carcinoma (ACC). Reproduction of TCGA ACC dataset showing (A) genetic alteration of a panel of adrenocortical hormone-related genes; (B) expression levels of a panel of adrenocortical hormone-related genes; (C) expression of HSD17B4 and its association with excessive hormonal type; (D) expression level of each gene grouped by the presence of excessive hormone; and (E) difference in overall survival between patients with or without HSD17B4 overexpression. 
tumor-suppressive role in ACC, and the effect was even cancer type restricted as the results we received using NCI-H295R cells could be only in part repeated in SW13 cells, which is not de facto ACC. Frequently altered genes towards amplified gene function (i.e. overexpression, focal amplification, etc.) do not necessary indicate protumorigenic role. This could result from selection-induced subclonal heterogeneity, and/or from treatment related response. Both notions can be perceived in our findings. First, high prevalence of overexpression yet lack of association with critical clinicopathological parameters (i.e. tumor stage, survival, etc.) indicates the existence of heterogeneity. Closer association with non-necrotic phenotype indicates such genotype towards better subtype of tumor. On the other hand, a near-significant association with adjuvant mitotane use is indicative for drug-related genetic response. In further in vitro studies, where microand macro- environments were much simpler, the tumor suppressive role of HSD17B4 was amplified.

In search of the potential mechanism thereof, we analyzed expressional correlation and enrichment for HSD17B4. Surprisingly, we captured substantial long list of significantly altered pathways from the KEGG pathway. We therefore carefully screened the pathways based on the recently published studies on genomic characterization of ACC $[20,21]$, including the TCGA ACC project whose data were used and reproduced in our study. Although the studies provide the most solid genomic data for this rare entity so far, it remains controversial how much those significantly mutated genes (SMGs) and related pathways substantially contribute to tumorigenesis and can be actionable. Taking the previous studies into account, current evidence supports TP53, CTNNB1, and PRKAR1A related signaling playing putative "driving" roles in ACC. Our finding that key elements in p53 signaling altered in HSD17B4 overexpressed cases support the tumor suppressive role we noted. Also, our findings that genetic manipulation of HSD17B4 did not alter cell motility in SW13 cells, indicating that effect of HSD17B4 is cell- and cancer- context dependent and constitutive HSD17B4 expression is important for its function. However, the interaction between p53 signaling and HSD17B4 remains in SW13 cells. The detailed interaction warrants further investigation.
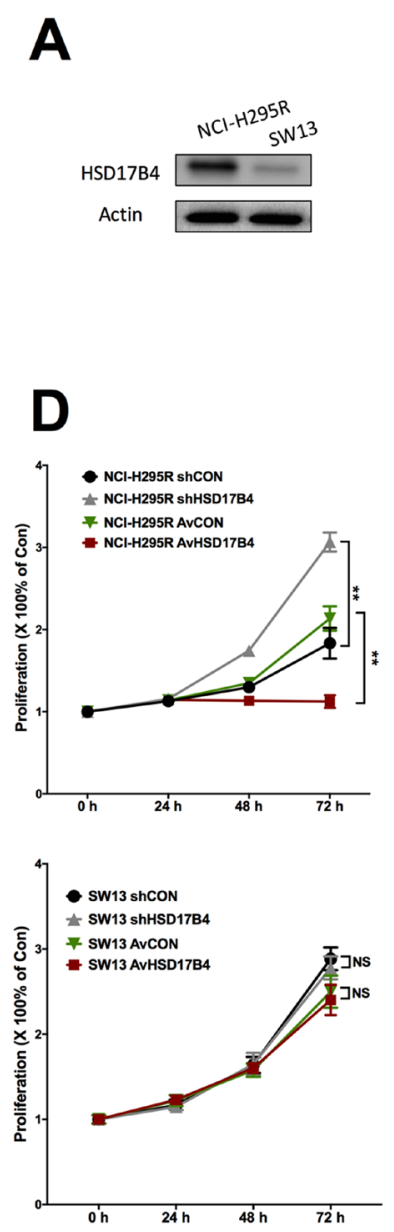
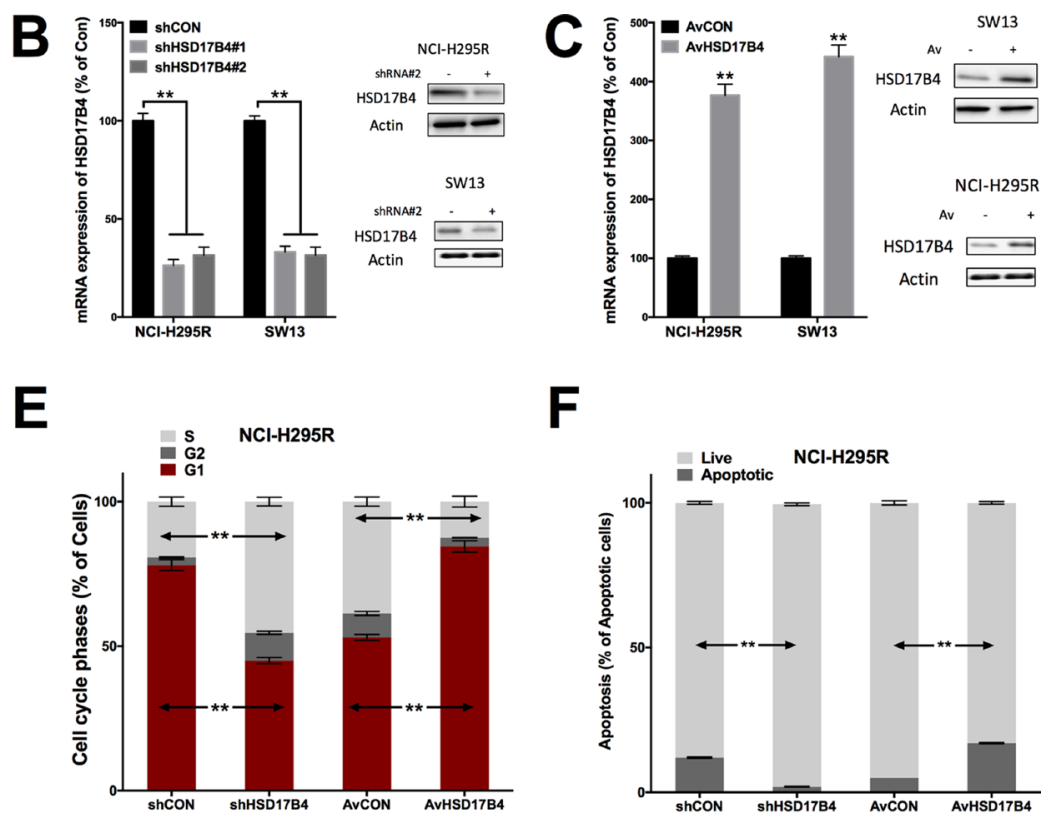

F
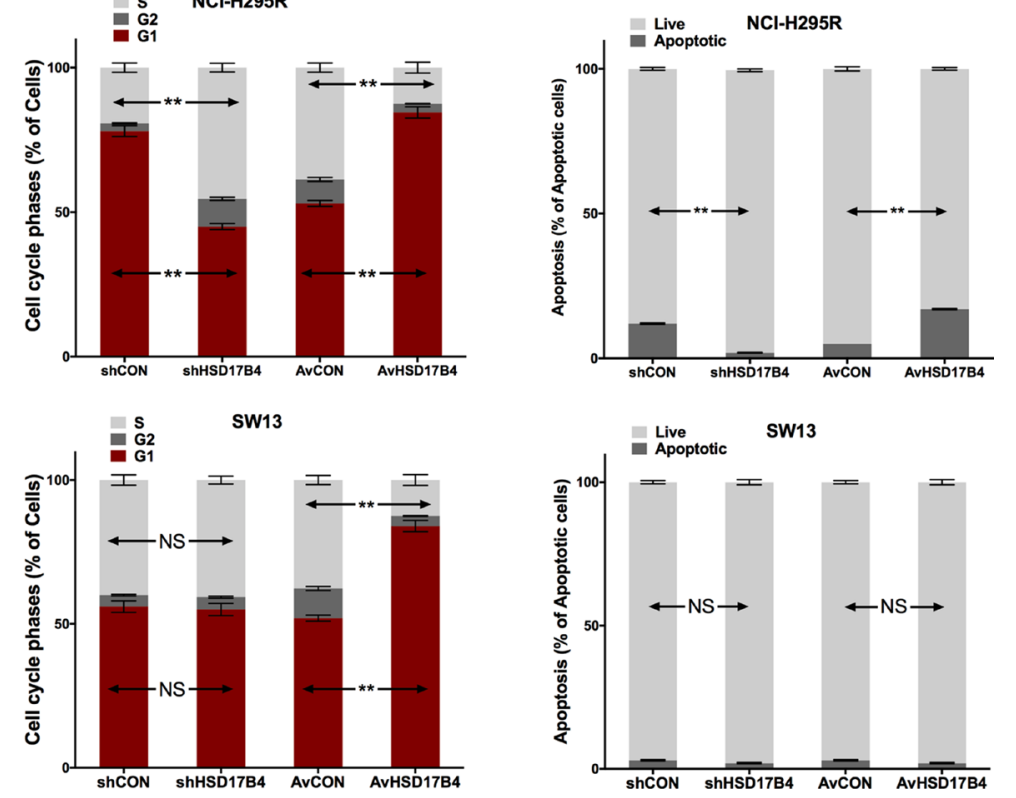

Figure 2: HSD17B4 exerts tumor suppressive effect in ACC cells NCI-H295R and SW13 showing. (A) constitutive HSD17B4 level; (B) robust knockdown (KD) of HSD17B4 using shRNAs in both cell lines and (C) robust upregulation of HSD17B4 in both cell lines; HSD17B4-KD and overexpression in NCI-H295 and SW13 cells effecting on (D) proliferation, (E) cell cycle and (F) cell apoptosis, respectively $\left({ }^{* *} P<0.01, n=3\right)$. 
Out study entails several unanswered questions. First, regulatory mechanism and biological effect of other enzymes altered at lower frequencies remains unstudied we are currently running studies thereon. Second, we did not investigate HSD17B4 expression in ACC cells treated with mitotane and other chemo-agents. This should be studied to evaluate the indicative role of HSD17B4 for treatment. Third, in the modern era, patient-derived cells lines can more accurately represent the current genetic spectrum than immortalized commercial cells. However, the success of establishment of patient-derived ACC cell line is opportunistic. Thus, in the current study, we used NCI-295R as the experimental model and SW13 as control. The currently available ACC cell for sale, or accessible for our group are exactly those 2 cells. Another cell line, the HAC15 cells available at ATCC (also the only ACC cell lines for sale at ATCC) is indeed a sub-clone of NCI-H295R. Last but not the least, the detailed regulatory mechanism between HSD17B4 and p53 signaling is still at large. We are now conducting mechanistic analysis on the molecular level, aiming to validate our observation.

\section{MATERIALS AND METHODS}

\section{Reproduction of TCGA-ACC dataset and functional annotation}

The cBioPortal online platform was used for reproduction of the TCGA-ACC database [22, 23], as previously reported [24]. We chose the dataset encompassing 92 samples and selected genomic profiles of mutations, putative copy-number alterations from GISTIC, and mRNA expression z-scores (RNA Seq V2 RSEM). We queried a series of genes that encoded key enzymes participating in adrenocortical steroid production $[25,26]$. Gene alterations were visualized using OncoPrint. By adding and sorting per clinical attribute of Excess Adrenal Hormone History Type, we generated expressional heatmap of the queried genes. Expression of a certain gene categorized by hormone type was visualized by the Plot function. Also, relative expression level of each gene in cases with or without hormone excess was analyzed. We then solely queried the gene status of HSD17B4.
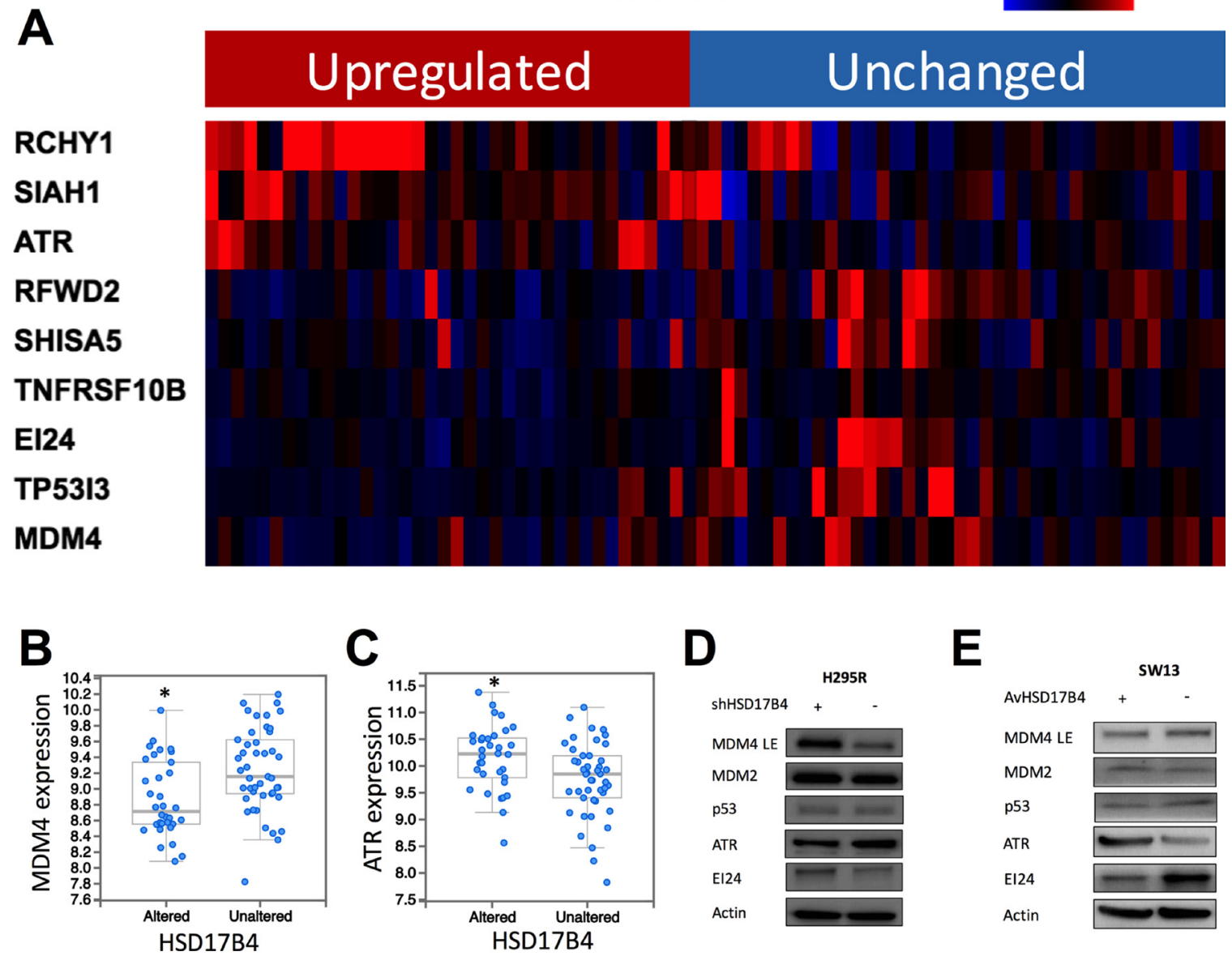

Figure 3: HSD17B4 overexpression is associated with p53 signaling alteration. (A) reproduction of TCGA ACC dataset showing heatmap of p53 signaling related genes in cases with and without HSD17B4 overexpression; (B) MDM4 expression is significantly lower in cases with HSD17B4 overexpression; (C) ATR expression is significantly higher in cases with HSD17B4 overexpression; enriched genes were confirmed for protein level using western blotting in (D) H295R cells with and without HSD17B4-KD, and in (E) SW13 cells with and without HSD17B4 replenish $\left({ }^{*} P<0.05\right)$. 
Alteration of HSD17B4, predominantly overexpression, was analyzed for association with clinicopathological parameters (adjuvant mitotane therapy, tumor stage, metastasis, Weiss score, response to primary therapy, residual tumor status, lymph node involvement, patient gender, and overall survival). We then extracted genes enriched amongst HSD17B4-altered and -unaltered cases. Significantly altered genes (passing both $p$ and $q$ values) were subject to the KOBAS 3.0 system for functional annotation and pathway analysis [27, 28]. The KEGG pathway database was used.

\section{Cell lines and culture}

The human adrenocortical cancer (NCI-H295R) and adrenocortical small cell carcinoma (SW13) cell lines were originally obtained from ATCC and cultured in DMEM medium (Thermo Scientific, Logan, UT, USA) supplemented with $20 \%$ fetal bovine serum.

\section{RNA interference}

Both cell lines were subject to HSD17B4 knockdown (KD) using shRNAs. The sequences for HSD17B4-KD were chosen from TRC (TRCN0000220382 for shRNA\#1 and TRCN0000220386 for shRNA\#2). Viral transduction was performed according to standard protocol [29]. Briefly, viral particles were prepared in 293T cells. Starved cells were transduced with equal titers of enveloped particles in OptiMEM media (Thermo) supplemented with $1 \mu \mathrm{g} / \mathrm{ml}$ polybrene (Sigma, Deisenhofen, Germany) for $24 \mathrm{~h}$. Puromycin was used for selection. Cells were also subject to HSD17B4 overexpression using adenovirus mediation. A human full-length cDNA clone of HSD17B4 on pCMV6-AC-GFP vector (Origene, Rockville, MD) was used and ligated to a secondary vector. After recombination procedure, HSD17B4-bearing and control adenovirus were generated. Cells infected were evaluated for multiplicities of infection (MOI) and cytopathy, and 100 MOI was designated as optimal.

\section{Quantitative RT-PCR}

Expression of HSD17B4 was quantified using Realtime RT-PCR, per established protocol [30]. Primers for HSD17B4 were as follows: Forward 5'- TGA GGG ATC GTT CCT TTG CTA -3'; Reverse 5'- CGT GTC ACT TGG AAT GAA CCC -3'. We used our in-house GAPDH primers for internal reference [30]. After conversion of extracted mRNA to cDNA, real-time PCR procedure with SYBR Green Premix Ex Taq (TaKaRa) in a $20-\mu \mathrm{L}$ system were run on ABI 7500n (Applied Biosystems, Forster City, CA). For each sample, the average value of threshold cycle was normalized to GAPDH level with the formula,

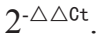

\section{Western blotting}

Standard western blotting procedure was followed [31]. Briefly, Cell lysates were separated with $12 \%$ sodium dodecyl sulfate polyacrylamide gel electrophoresis and transferred onto nitrocellulose membranes, which were subsequently blocked for $4 \mathrm{hrs}$. Membranes were then incubated with non-fat milk-diluted primary antibodies: Rabbit polyclonal anti-HSD17B4 (Sigma); mouse monoclonal antibody against MDM2 (Abcam, Cambridge,
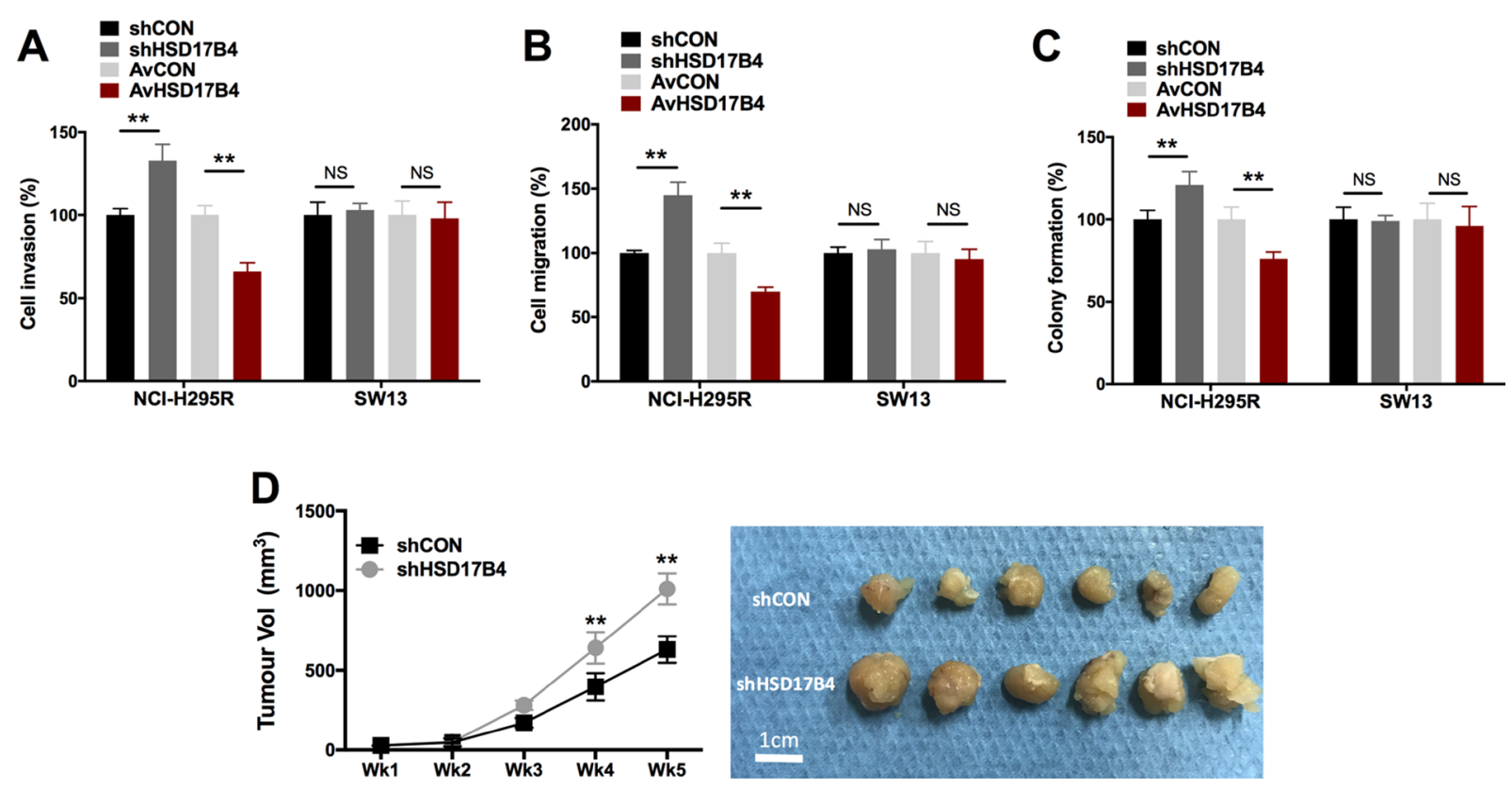

Figure 4: HSD17B4 altered cell motility in H295R but not in SW13 cells. HSD17B4-KD and overexpression in NCI-H295 and SW13 cells effecting on (A) cell invasion; (B) cell migration; and (C) colony formation, respectively; (D) HSD17B4-KD induced increased in vivo tumor growth of NCI-H295R cells $\left({ }^{* *} P<0.01\right.$, NS = not significant, $\left.n=3\right)$. 
UK); Anti-MDM4 TRUEMAB antibody (Origene); anti-p53 mouse monoclonal antibody (Abcam); rabbit polyclonal antibody against ATR (Abcam); and rabbit polyclonal anti-IE24 antibody (Abcam). Corresponding secondary antibodies were applied followed by electrochemiluminescence (ECL) processing. MDM4 was subject to long exposure (LE) due to relatively weak signal.

\section{Proliferation assay}

The crystal violet assay was used to evaluate cell proliferation. Cells were seeded in 96-well plates at a density of 2500 cells/well for $0,24,48$ and $72 \mathrm{~h}$. At each time point, medium was gently removed and cells were fixed with formalin. Cells were then stained with $0.05 \% \mathrm{CV}$ for $30 \mathrm{~min}$. Methanol was applied and plates were read at absorbance of OD $540 \mathrm{~nm}$. All readings were normalized to $0 \mathrm{~h}$ status.

\section{Cell cycle and apoptosis}

The cell cycle and apoptosis in the current study were measured using flow cytometry. For cell cycle analysis, cells were trypsinized and treated with cell cycle staining buffer (MultiSciences Biotech, Hangzhou, China) for $15 \mathrm{~min}$. Suspension were then subject to flow cytometry on a BD FACSCanto flow cytometer. For apoptosis, cells were stained with Annexin V-fluorescein (BD Pharmingen, Pasig City, Philippines) and propidium iodide (PI) (BD) for $15 \mathrm{~min}$ at room temperature. Samples were then analyzed with flow cytometry to determine percentages of apoptotic cells using Annexin V/PI indication.

\section{Migration, invasion and colony formation}

Both migration and invasion were studied with Transwell assays. Roughly 1000 cells were cultured in the Transwell inserts either coated (for invasion) or uncoated (for migration) with Matrigel. The inserts were placed in a 24-well plate filled with complete medium. Cells that penetrated to the underside surfaces of the inserts were fixed and stained with the crystal violet. The mean of cell number of three high power fields for each condition was calculated. Colony formation assay was used to profile anchorage-independent growth. Briefly, 1000 cells were seeded in the mixture of $10 \%$ FBS-containing medium and $0.4 \%$ agarose, which was layered on top of $0.6 \%$ agar in $20 \% \mathrm{FBS}$-containing medium. On top of the agarose was $1 \mathrm{ml}$ of complete medium that was changed every 3 days. Two weeks later, the plates were stained with $0.005 \%$ of crystal violet for $1 \mathrm{~h}$. Colonies were counted microscopically.

\section{Xenograft model}

Twenty male athymic nude mice at 6 weeks of age were bred in SPF (special pathogen-free) grade laboratory.
Mice were randomly divided into 2 groups (Treatment versus control). A total of $1.5 \times 10^{7} \mathrm{NCI}-\mathrm{H} 295 \mathrm{R}$ cancer cells with or without HAD17B4-KD, resuspended in 100 $\mathrm{ml}$ of PBS were injected subcutaneously at the left axilla of each mouse. Mice were monitored every 3 days for general condition and tumor growth and all were sacrificed on Day. Tumor size was calculated with the formula, Length $\times$ Width $^{2} \times 0.5236$.

\section{Statistical analysis}

All data were processed with the SPSS ver.21 and Prism Graphpad ver.6 software. For comparison between means of 2 cohorts, the Student's $t$-test was used. For survival analysis, the Kaplan Meier plot and Log rank test were used. Correlation was studied using the Spearman test. Enrichment analyses were automatically computed by the cBioPortal platform and KOBAS 3.0 system. All data were presented as mean \pm standard deviation (SD). All in vitro studies were performed in triplicates with 3 replications. The $P$ value of $<.05$ was accepted as statistically significant and the $Q$ value of $<.05$ was accepted for the threshold of false discovery rate (FDR).

\section{CONCLUSIONS}

ACC is a rare but aggressive disease. It is often associated with excessive hormone production. We find adrenocortical hormone related gene HSD17B4 is overexpressed at high frequency in ACC but it is not associated with hormone excess. Overexpression of HSD17B4 exerts tumor suppressive role in ACC and it may play a role in p53 signaling.

\section{CONFLICTS OF INTEREST}

None.

\section{FUNDING}

This study was supported in part by Youth Elites Scientific Funding of Shanghai Municipal Health Bureau (Grant No.20144Y0111) and General Program Scientific Funding of Shanghai Municipal Health Bureau (Grant No.201440557).

\section{REFERENCES}

1. Bilimoria KY, Shen WT, Elaraj D, Bentrem DJ, Winchester DJ, Kebebew E, Sturgeon C. Adrenocortical carcinoma in the United States: treatment utilization and prognostic factors. Cancer. 2008; 113:3130-3136.

2. Else T, Giordano TJ, Hammer GD. Evaluation of telomere length maintenance mechanisms in adrenocortical carcinoma. J Clin Endocrinol Metab. 2008; 93:1442-1449. 
3. Else T, Williams AR, Sabolch A, Jolly S, Miller BS, Hammer GD. Adjuvant therapies and patient and tumor characteristics associated with survival of adult patients with adrenocortical carcinoma. J Clin Endocrinol Metab. 2014; 99:455-461.

4. Fassnacht M, Johanssen S, Quinkler M, Bucsky P, Willenberg HS, Beuschlein F, Terzolo M, Mueller HH, Hahner S, Allolio B, and German Adrenocortical Carcinoma Registry Group, and European Network for the Study of Adrenal Tumors. Limited prognostic value of the 2004 International Union Against Cancer staging classification for adrenocortical carcinoma: proposal for a Revised TNM Classification. Cancer. 2009; 115:243-250.

5. Fassnacht M, Kroiss M, Allolio B. Update in adrenocortical carcinoma. J Clin Endocrinol Metab. 2013; 98:4551-4564.

6. Calissendorff J, Calissendorff F, Falhammar H. Adrenocortical cancer: mortality, hormone secretion, proliferation and urine steroids - experience from a single centre spanning three decades. BMC Endocr Disord. 2016; 16:15.

7. Trevisan M, Matkovic U, Cusinato R, Toppo S, Palu G, Barzon L. Human cytomegalovirus productively infects adrenocortical cells and induces an early cortisol response. J Cell Physiol. 2009; 221:629-641.

8. Barzon L, Trevisan M, Masi G, Pacenti M, Sinigaglia A, Macchi V, Porzionato A, De Caro R, Favia G, Iacobone M, Palu G. Detection of polyomaviruses and herpesviruses in human adrenal tumors. Oncogene. 2008; 27:857-864.

9. Cheungpasitporn W, Horne JM, Howarth CB. Adrenocortical carcinoma presenting as varicocele and renal vein thrombosis: a case report. J Med Case Rep. 2011; 5:337.

10. Zheng S, Cherniack AD, Dewal N, Moffitt RA, Danilova L, Murray BA, Lerario AM, Else T, Knijnenburg TA, Ciriello G, Kim S, Assie G, Morozova O, et al. Comprehensive PanGenomic Characterization of Adrenocortical Carcinoma. Cancer Cell. 2016; 30:363.

11. Gilep AA, Sushko TA, Usanov SA. At the crossroads ofsteroid hormone biosynthesis: the role, substrate specificity and evolutionary development of CYP17. Biochim Biophys Acta. 2011; 1814:200-209.

12. Chung BC, Picado-Leonard J, Haniu M, Bienkowski M, Hall PF, Shively JE, Miller WL. Cytochrome P450c17 (steroid 17 alpha-hydroxylase/17,20 lyase): cloning of human adrenal and testis cDNAs indicates the same gene is expressed in both tissues. Proc Natl Acad Sci USA. 1987; 84:407-411.

13. Miller WL. Minireview: regulation of steroidogenesis by electron transfer. Endocrinology. 2005; 146:2544-2550.

14. Staels B, Hum DW, Miller WL. Regulation of steroidogenesis in NCI-H295 cells: a cellular model of the human fetal adrenal. Mol Endocrinol. 1993; 7:423-433.

15. Payabyab EC, Balasubramaniam S, Edgerly M, Velarde M, Merino MJ, Venkatesan AM, Leuva H, Litman T, Bates SE, Fojo T. Adrenocortical Cancer: A Molecularly Complex Disease Where Surgery Matters. Clin Cancer Res. 2016; 22:4989-5000.
16. Choi YM, Kwon H, Jeon MJ, Sung TY, Hong SJ, Kim TY, Kim WB, Shong YK, Lee JL, Song DE, Kim WG. Clinicopathological Features Associated With the Prognosis of Patients With Adrenal Cortical Carcinoma: Usefulness of the Ki-67 Index. Medicine (Baltimore). 2016; 95:e3736.

17. Berruti A, Baudin E, Gelderblom H, Haak HR, Porpiglia F, Fassnacht M, Pentheroudakis G, Group EGW. Adrenal cancer: ESMO Clinical Practice Guidelines for diagnosis, treatment and follow-up. Ann Oncol. 2012; 23 7:vii131-138.

18. Rasiah KK, Gardiner-Garden M, Padilla EJ, Moller G, Kench JG, Alles MC, Eggleton SA, Stricker PD, Adamski J, Sutherland RL, Henshall SM, Hayes VM. HSD17B4 overexpression, an independent biomarker of poor patient outcome in prostate cancer. Mol Cell Endocrinol. 2009; 301:89-96.

19. Lu X, Ma P, Shi Y, Yao M, Hou L, Zhang P, Jiang L. NFkappaB increased expression of 17beta-hydroxysteroid dehydrogenase 4 promotes HepG2 proliferation via inactivating estradiol. Mol Cell Endocrinol. 2015; 401:1-11.

20. Assie G, Letouze E, Fassnacht M, Jouinot A, Luscap W, Barreau O, Omeiri H, Rodriguez S, Perlemoine K, RenéCorail F, Elarouci N, Sbiera S, Kroiss M, et al. Integrated genomic characterization of adrenocortical carcinoma. Nat Genet. 2014; 46:607-612.

21. Zheng S, Cherniack AD, Dewal N, Moffitt RA, Danilova L, Murray BA, Lerario AM, Else T, Knijnenburg TA, Ciriello G, Kim S, Assie G, Morozova O, et al. Comprehensive PanGenomic Characterization of Adrenocortical Carcinoma. Cancer Cell. 2016; 29:723-736.

22. Cerami E, Gao J, Dogrusoz U, Gross BE, Sumer SO, Aksoy BA, Jacobsen A, Byrne CJ, Heuer ML, Larsson E, Antipin Y, Reva B, Goldberg AP, et al. The cBio cancer genomics portal: an open platform for exploring multidimensional cancer genomics data. Cancer Discov. 2012; 2:401-404.

23. Gao J, Aksoy BA, Dogrusoz U, Dresdner G, Gross B, Sumer SO, Sun Y, Jacobsen A, Sinha R, Larsson E, Cerami $\mathrm{E}$, Sander C, Schultz N. Integrative analysis of complex cancer genomics and clinical profiles using the cBioPortal. Sci Signal. 2013; 6:p11.

24. Feng C, Sun Y, Ding G, Wu Z, Jiang H, Wang L, Ding Q, Wen H. PI3Kbeta inhibitor TGX221 selectively inhibits renal cell carcinoma cells with both VHL and SETD2 mutations and links multiple pathways. Sci Rep. 2015; 5:9465.

25. Else T, Kim AC, Sabolch A, Raymond VM, Kandathil A, Caoili EM, Jolly S, Miller BS, Giordano TJ, Hammer GD. Adrenocortical carcinoma. Endocr Rev. 2014; 35:282-326.

26. Fiorentini C, Fragni M, Perego P, Vezzoli S, Bonini SA, Tortoreto M, Galli D, Claps M, Tiberio GA, Terzolo M, Missale C, Memo M, Procopio G, et al. Antisecretive and Antitumor Activity of Abiraterone Acetate in Human Adrenocortical Cancer: A Preclinical Study. J Clin Endocrinol Metab. 2016; 101:4594-4602.

27. Xie C, Mao X, Huang J, Ding Y, Wu J, Dong S, Kong L, Gao G, Li CY, Wei L. KOBAS 2.0: a web server for 
annotation and identification of enriched pathways and diseases. Nucleic Acids Res. 2011; 39:W316-322.

28. Wu J, Mao X, Cai T, Luo J, Wei L. KOBAS server: a webbased platform for automated annotation and pathway identification. Nucleic Acids Res. 2006; 34:W720-724.

29. Feng C, Ding G, Jiang H, Ding Q, Wen H. Loss of MLH1 confers resistance to PI3Kbeta inhibitors in renal clear cell carcinoma with SETD2 mutation. Tumour Biol. 2015; $36: 3457-3464$.
30. Wen H, Feng CC, Ding GX, Meng DL, Ding Q, Fang ZJ, Xia GW, Xu G, Jiang HW. Med19 promotes bone metastasis and invasiveness of bladder urothelial carcinoma via bone morphogenetic protein 2. Ann Diagn Pathol. 2013; 17:259-264.

31. Feng C, Ho Y, Sun C, Xia G, Ding Q, Gu B. TFPI-2 expression is decreased in bladder cancer and is related to apoptosis. J BUON. 2016; 21:1518-1523. 